\title{
Two new cocrystals of lanthanide aquo complexes with aromatic molecules
}

Bulat Gabidullin, Yixin Zhang, Amélie Pialat, Muralee Murugesu

University of Ottawa, Ottawa, ON, Canada, bulatgabidullin@gmail.com

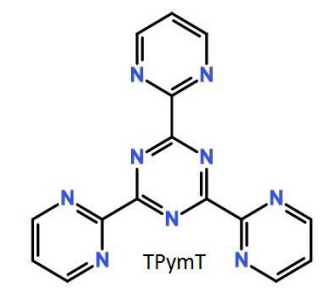<smiles>c1cc(-c2nccnn2)nc(-c2nccnn2)c1</smiles>

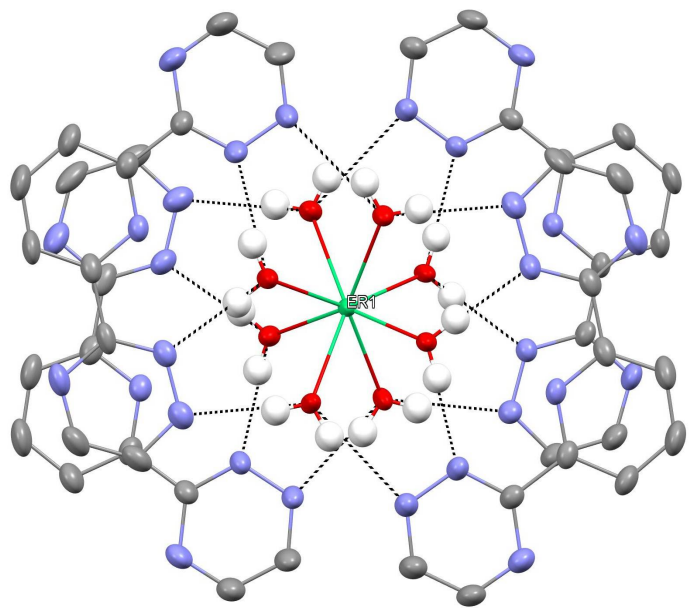

The crystal structure is reported for $\left[\mathrm{Er}\left(\mathrm{H}_{2} \mathrm{O}\right)_{8}\right] \cdot 4 \mathrm{btp} \cdot 6 \mathrm{H}_{2} \mathrm{O} \cdot 3 \mathrm{Cl}(\mathbf{1})$ and $2\left[\mathrm{La}\left(\mathrm{H}_{2} \mathrm{O}\right)_{8}(\mathrm{MeCN})\right] \cdot$ -3TPymT $\cdot 7 \mathrm{H}_{2} \mathrm{O} \cdot 6 \mathrm{ClO}_{4}(2)$, where the salts of aquo and acetonitrile-aquo lanthanide complexes cocrystallize with planar aromatic molecules. In $\mathbf{1}$ the octaaqua-erbium complex has a square antiprismatic geometry; it is elegantly surrounded by four btp ligands and bonded with them by O-H..N H-bonds. The resulting flat molecular aggregates are stacked in the crystal parallel to the (11-1) plane. The chloride counter-anions and non-coordinating water molecules occupy space between those aggregates; they are interconnected by H-bonds and form perforated layers parallel to the (001) plane. In 2 the acetonitrile-octaaqua-lathanum complex has a tricapped trigonal prismatic geometry. Its hydrogen bond partners are not limited to TPymT and include water molecules and perchlorate anions, probably because the TPymT ligand lacks nitrogen atoms to act similarly to btp in $\mathbf{1}$. Together with acetonitrile coordinated to $\mathrm{La}$ and bulkiness of perchlorate anions, this leads to disturbance in molecular packing, and the TPymT molecules barely have a common orientation in the crystal; the plane of the molecules deviates by $\pm 27^{\circ}$ and $\pm 29^{\circ}$ from the (001) crystallographic plane. 